\title{
Pirfenidone in idiopathic pulmonary fibrosis
}

\author{
H. Taniguchi*, M. Ebina*, Y. Kondoh*, T. Ogurađ, A. Azuma ${ }^{+}$, M. Suga $^{\S}$, Y. Taguchi' ${ }^{f}$, \\ H. Takahashi**, K. Nakata ${ }^{\# \#}$, A. Sato ${ }^{\uparrow \uparrow}$, M. Takeuchi ${ }^{++}$, G. Raghu ${ }^{\S \S}$, S. Kudoh ${ }^{+}$and \\ T. Nukiwa ${ }^{\#}$, and the Pirfenidone Clinical Study Group in Japan ${ }^{f f}$
}

ABSTRACT: Idiopathic pulmonary fibrosis (IPF) is a progressive lung disease without proven effective therapy.

A multicentre, double-blind, placebo-controlled, randomised phase III clinical trial was conducted in Japanese patients with well-defined IPF to determine the efficacy and safety of pirfenidone, a novel antifibrotic oral agent, over 52 weeks. Of 275 patients randomised (high-dose, $1,800 \mathrm{mg} \cdot$ day $^{-1}$; low-dose, $1,200 \mathrm{mg} \cdot \mathrm{day}^{-1}$; or placebo groups in the ratio 2:1:2), 267 patients were evaluated for the efficacy of pirfenidone. Prior to unblinding, the primary end-point was revised; the change in vital capacity (VC) was assessed at week 52. Secondary end-points included the progression-free survival (PFS) time.

Significant differences were observed in VC decline (primary end-point) between the placebo group $(-0.16 L)$ and the high-dose group $(-0.09 L)(p=0.0416)$; differences between the two groups $(p=0.0280)$ were also observed in the PFS (the secondary end-point). Although photosensitivity, a well-established side-effect of pirfenidone, was the major adverse event in this study, it was mild in severity in most of the patients.

Pirfenidone was relatively well tolerated in patients with IPF. Treatment with pirfenidone may decrease the rate of decline in VC and may increase the PFS time over 52 weeks. Additional studies are needed to confirm these findings.

KEYWORDS: Idiopathic pulmonary fibrosis, pirfenidone, progression-free survival time, vital capacity

然

diopathic pulmonary fibrosis (IPF) is a devastating, progressive fibrotic lung disease with a median survival of 3-5 yrs without proven effective therapy $[1,2]$. Recent studies have suggested that IPF develops from chronic epithelial cell injury and aberrant activation of progressive fibrosis $[3,4]$. Therefore, the therapeutic strategy against IPF has shifted from corticosteroids and/or immunosuppressants to antifibrotic agents, as reported in recent clinical trials $[5,6]$.

Pirfenidone (5-methyl-1-phenyl-2-[1H]-pyridone; Shionogi \& Co., Osaka, Japan; MARNAC, Dallas, TX, USA) [7-9] is a promising agent with therapeutic potential for IPF that has combined anti-inflammatory, antioxidant and antifibrotic effects in experimental models of pulmonary fibrosis [10-14]. Following an open-label phase II pioneer study [7] and an open-label 1-yr study in Japan [9], a double-blind, placebo-controlled clinical trial of pirfenidone in Japanese patients with IPF demonstrated a lesser decline of vital capacity $(\mathrm{VC})$ in patients receiving pirfenidone for

For editorial comments see page 728 .
9 months [15]. The trial was prematurely terminated by the independent Data and Safety Monitoring Board (DSMB) because of a higher incidence of acute exacerbations in the placebo group than the pirfenidone group. These encouraging results, prompted us to undertake a phase III 1-yr clinical study to examine the therapeutic effects of pirfenidone on lung functional deterioration and disease progression in patients with IPF.

\section{MATERIALS AND METHODS}

\section{Study subjects}

The diagnosis of IPF was in accordance with the American Thoracic Society (ATS)/European Respiratory Society (ERS) Consensus statement [16] and the fourth version of the clinical diagnostic criteria guidelines for idiopathic interstitial pneumonia in Japan [17]. High-resolution computed tomography (HRCT) scans of the chest were reviewed by expert chest radiologists prior to randomisation; two out of six expert radiologists independently evaluated the HRCT images to agree and determine whether the pattern of usual interstitial pneumonia (UIP) was present or not in accordance with the predetermined protocol (see online supplementary material). In cases
AFFILIATIONS

*Dept of Respiratory Medicine and Allergy, Tosei General Hospital, Aichi,

"Dept of Respiratory Medicine Tohoku University Graduate School of Medicine, Sendai,

"Dept of Respiratory Medicine, Kanagawa Cardiovascular and Respiratory Center, Yokohama, +Fourth Dept of Internal Medicine, Nippon Medical School, Tokyo, \#\# Nakata clinic, Tokyo, ${ }^{+}$Dept of Biostatistics, Kitasato University, Tokyo,

${ }^{\S}$ Dept of Respiratory Medicine, Saiseikai Kumamoto Hospital, Kumamoto,

${ }^{f}$ Dept of Respiratory Medicine, Tenri Hospital, Tenri,

**Third Dept of Internal Medicine,

Sapporo Medical University Hospital Sapporo, and

" Kyoto Preventive Medical Center, Kyoto, Japan.

${ }^{\S \S}$ University of Washington Medical Center, Seattle, WA, USA.

${ }^{f f} \mathrm{~A}$ full list of the members of the Pirfenidone Clinical Study Group in Japan and their affiliations can be found in the Acknowledgements section.

CORRESPONDENCE

T. Nukiwa,

Dept of Respiratory Medicine

Tohoku University Graduate School of Medicine,

Sendai 980-8574,

Japan

E-mail: toshinkw@idac.tohoku.ac.jp

Received:

Jan 132009

Accepted after revision: Nov 202009

First published online:

Dec 082009

European Respiratory Journal Print ISSN 0903-1936 Online ISSN 1399-3003 
of disagreement, the interpretation of the third radiologist influenced the final decision, and the diagnosis of patients with probable UIP pattern on HRCT was confirmed by the presence of histopathological UIP pattern in surgical lung biopsy samples.

Eligible patients were adults (20-75 yrs of age) with IPF diagnosis based on the above criteria and meeting the following arterial oxygen saturation measured by pulse oximetry $\left(\mathrm{Sp}, \mathrm{O}_{2}\right)$ criteria: 1) oxygen desaturation of $\geqslant 5 \%$ difference between resting $\mathrm{Sp}_{\mathrm{O}} \mathrm{O}_{2}$ and the lowest $\mathrm{Sp}, \mathrm{O}_{2}$ during a 6-min steady-state exercise test (6MET); and 2) the lowest $\mathrm{Sp}_{\mathrm{p}} \mathrm{O}_{2}$ during the $6 \mathrm{MET}$ of $\geqslant 85 \%$ while breathing air. The $6 \mathrm{MET}$ procedure was in accordance with previous study protocols (see online supplementary material). Exclusion criteria were: 1) a decrease in symptoms during the preceding 6 months; 2) use of immunosuppressants and/or oral corticosteroids at a dose $>10 \mathrm{mg} \cdot \mathrm{day}^{-1}$ during the preceding 3 months; 3 ) clinical features of idiopathic interstitial pneumonia other than IPF; 4) evidence of known coexisting pulmonary hypertension, asthma, tuberculosis, bronchiectasis, aspergillosis or severe respiratory infection.

The protocol was approved by the institutional review board at each centre and written informed consent was obtained from all participants prior to enrolment. The ongoing efficacy and safety results were reviewed by the independent DSMB.

\section{Study design}

This study was a multicentre, double-blind, randomised, placebo-controlled, phase III clinical trial designed to determine the efficacy and safety of oral administration of pirfenidone for $1 \mathrm{yr}$ in patients with IPF. Eligible patients were allocated to three groups: high dose $\left(1,800 \mathrm{mg} \cdot\right.$ day $\left.^{-1}\right)$, low dose $\left(1,200 \mathrm{mg} \cdot \mathrm{day}^{-1}\right)$ and placebo, in a ratio of 2:1:2, respectively, with a modified minimisation method, including some random allocation based on biased coin design to balance baseline $S_{\mathrm{p}}, \mathrm{O}_{2}[18,19]$.

\section{Treatment regimen}

Pirfenidone as 200-mg tablets and matching placebo were provided for oral use by Shionogi \& Co. The dose was increased in a stepwise manner as follows: one tablet t.i.d. orally administered for the first 2 weeks (high dose $600 \mathrm{mg} \cdot$ day $^{-1}$, low dose $600 \mathrm{mg} \cdot$ day $^{-1}$ and placebo $0 \mathrm{mg} \cdot$ day $^{-1}$ ), then two tablets per dose t.i.d. for the following 2 weeks (high dose $1,200 \mathrm{mg} \cdot \mathrm{day}^{-1}$, low dose $600 \mathrm{mg} \cdot \mathrm{day}^{-1}$ and placebo $0 \mathrm{mg} \cdot \mathrm{day}^{-1}$ ) and three tablets t.i.d. for the remaining 48 weeks (high dose 1,800 mg $\cdot$ day $^{-1}$, low dose 1,200 mg.day ${ }^{-1}$ and placebo $0 \mathrm{mg}^{-\mathrm{day}^{-1}}$ ) (see online supplementary material). Although concomitant use of corticosteroid $\leqslant 10 \mathrm{mg} \cdot$ day $^{-1}$ (as the prednisone equivalent) was permitted during the study period, concomitant use of immunosuppressants and other experimental agents under investigation was not allowed (see online supplementary material). All participants were forewarned of the potential photosensitivity skin rash and were advised to use sunscreens during exposure to direct sunlight.

\section{Efficacy end-points}

The primary end-point was the change in VC from baseline to week 52. Secondary end-points were progression-free survival (PFS) time and the change in the lowest $\mathrm{Sp}_{\mathrm{O}} \mathrm{O}_{2}$ during the $6 \mathrm{MET}$. The progression of disease was defined by death and/or $\geqslant 10 \%$ decline in VC from baseline. When the VC data could not be obtained due to worsening of respiratory symptoms, including acute exacerbation, the case was also classified as disease progression. As in the phase II study conducted in Japan [15], the $6 \mathrm{MET}$ procedure had been prespecified in the protocol (see online supplementary material). Tertiary end-points were pulmonary function tests (PFTs; arterial oxygen tension, alveolar-arterial oxygen tension difference at rest, total lung capacity (TLC) and diffusing capacity of the lung for carbon monoxide $(D L, C O))$, acute exacerbation [20], serum levels of the markers of interstitial pneumonias (sialylated carbohydrate antigen KL-6, surfactant protein (SP)-D and SP-A; see online supplementary material) and subjective/objective symptoms (cough, presence/absence of sputum and dyspnoea in daily living assessed with Hugh-Jones classification).

VC was measured every 4 weeks, whereas the lowest $S \mathrm{p}, \mathrm{O}_{2}$ during the 6MET and other PFTs were determined every 12 weeks. Acute exacerbation of IPF was defined according to previous reports and revised criteria for acute exacerbation of IPF in Japan (see appendices in online supplementary material) $[15,20]$.

The change in the lowest $S_{\mathrm{p}}, \mathrm{O}_{2}$ during the $6 \mathrm{MET}$ over 52 weeks was the original intended primary end-point for this study. The decision was made to revise the primary end-point from the lowest $\mathrm{Sp}, \mathrm{O}_{2}$ to $\mathrm{VC}$ at week 52 and assess the change in the lowest $\mathrm{Sp}_{\mathrm{p}} \mathrm{O}_{2}$ during the $6 \mathrm{MET}$ as a secondary end-point. This was recommended by the independent DSMB, prior to breaking the code. This decision was based on the evolved knowledge of assessment with objective measurements in IPF [21-26], as well as the lack of validation in the 6MET study (unpublished data) and difficulties in reproducibility of the $\mathrm{Sp}, \mathrm{O}_{2}$ measurements during the 6-min walk test (6MWT) [27].

\section{Statistical analysis}

The planned sample size was 250 in total: 100, 50 and 100 patients in the high-dose, low-dose and placebo groups, respectively. The sample sizes of 100 for the high-dose and placebo groups were determined on the basis of simulations that would provide a statistical power of 0.8 to detect assumed differences of the mean changes in the lowest $\mathrm{Sp}, \mathrm{O}_{2}$ from baseline to week 52 between the two groups at a significance level in this study of 0.1 (two-sided) (details in the online supplementary material). Although the primary end-point was altered from the lowest $\mathrm{Sp}, \mathrm{O}_{2}$ to $\mathrm{VC}$ after the study was started, the power calculated on the basis of the change in VC turned out to be the same (maintained at $\sim 0.8$ ) and, thus, the planned sample size was not altered. As the low-dose group was intended to assess benefit-risk profiles of pirfenidone treatment at a tapered dose, the sample size of the low-dose group was obtained by halving the sample size of the high-dose and placebo groups. Multiplicity problems were not taken into account because the main analysis was the comparison between the high-dose and placebo groups.

Analyses of the change in $\mathrm{VC}$ and the lowest $S \mathrm{p}, \mathrm{O}_{2}$ from baseline were performed with ANCOVA using the respective baseline measurements as covariates. Analyses of the change in other PFTs and the serum levels of the markers of interstitial pneumonias were performed with the least significant difference method based on one-way ANOVA. The principle of the 
last observation carried forward (LOCF) was adopted to impute missing values if patient data were available for $\geqslant 4$ weeks after the baseline. In order to avoid bias when dealing with missing values, the mixed model approach using the available repeated measures of changes in VC was performed as a sensitivity analysis. The cumulative PFS rates were estimated using the Kaplan-Meier method and compared using the log-rank test. Incidences were compared with Fisher's exact test.

\section{RESULTS}

\section{Patients enrolled}

Between July 2004 and August 2005, 325 patients were screened at 73 centres in Japan, and 275 patients were randomised to one of the three groups: high dose, low dose and placebo. Of the 275 patients, 267 (108, 55 and 104 patients in the high-dose, low-dose and placebo groups, respectively) were deemed eligible for the full analysis set. Eight patients were excluded because no post-baseline data were available (fig. 1). The first patient entered the trial on July 13, 2004, and the last patient entered on August 30, 2005.

No significant differences were seen in the distribution of the demographic and baseline characteristics among the three groups, except for smoking history (table 1). A post analysis did not reveal a significant effect of smoking history on the change of VC. Based on their PFTs, patients had been assumed to have relatively mild functional impairment. 246 patients
(92\%) had not received prior treatment for IPF, including corticosteroids. 86 patients $(40,15$ and 31 patients in the highdose, low-dose and placebo groups, respectively) discontinued the study medication for various reasons (table 2). The main reasons were progression of disease in the placebo group, and the occurrence of adverse events in both pirfenidone treatment groups. A Kaplan-Meier plot of the time to discontinuation for the three groups is shown in Figure E1 of the online supplementary material. The time distributions were compared in pairs among the three groups using the log-rank test, but no significant differences were seen. 11 patients $(4.1 \%)$ died during the study: three, four and four in the high-dose, lowdose and placebo groups, respectively.

\section{Effects on primary end-points}

The adjusted means of the changes in VC based on the ANCOVA were $-0.09 \mathrm{~L}$ and $-0.16 \mathrm{~L}$ in the high-dose and placebo groups, respectively, with a difference of $0.07 \mathrm{~L}$ being significant $(p=0.0416)$. In addition, the adjusted mean change in the low-dose group was $-0.08 \mathrm{~L}$; a significant difference was also seen between the low-dose and placebo groups $(p=0.0394)$ (fig. 2). The crude means (at baseline and week 52) and the changes, the adjusted means, mean changes from ANCOVA and the p-values are summarised in table 3 . No significant difference was seen between the high- and low-dose groups. The serial changes in VC over the 52-week period are illustrated in figure 3.

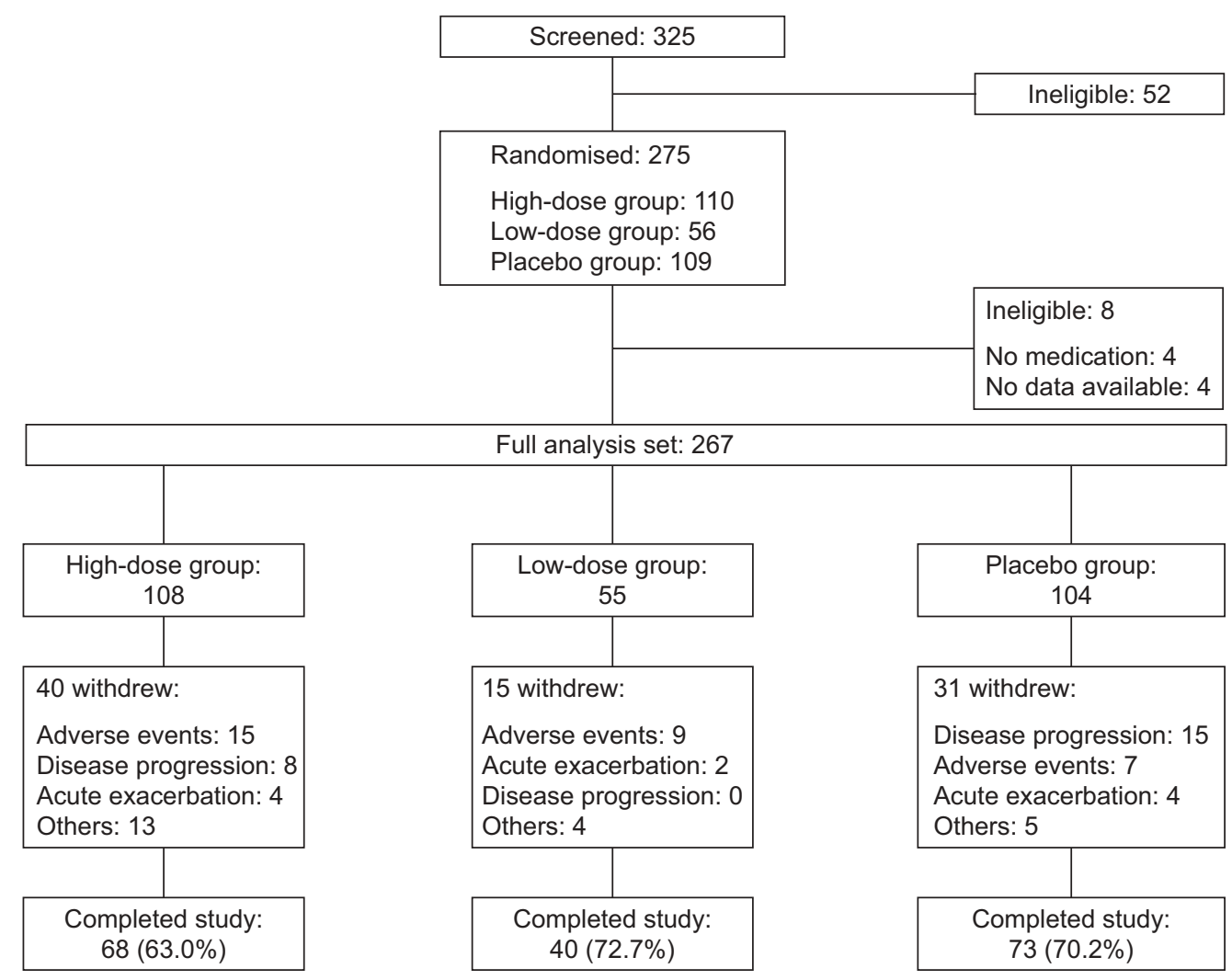

FIGURE 1. Disposition of patients. 325 patients were screened at 73 centres in Japan and 275 patients were randomised to one of the three groups: high-dose $\left(1,800 \mathrm{mg} \cdot\right.$ day $^{-1}$ of pirfenidone), low-dose $\left(1,200 \mathrm{mg} \cdot\right.$ day $\left.^{-1}\right)$ and placebo groups. Disease progression included a $10 \%$ decline in vital capacity and worsening of respiratory symptoms. 


\begin{tabular}{|c|c|c|c|c|}
\hline Male & $85(78.7)$ & $47(85.5)$ & $81(77.9)$ & 0.53 \\
\hline Female & $23(21.3)$ & $8(14.5)$ & $23(22.1)$ & \\
\hline Age yrs & $65.4 \pm 6.2$ & $63.9 \pm 7.5$ & $64.7 \pm 7.3$ & 0.44 \\
\hline Smokers & $5(4.6)$ & $10(18.2)$ & $13(12.5)$ & $0.07^{\circ}$ \\
\hline Ex-smokers & $81(75.0)$ & $33(60.0)$ & $70(67.3)$ & \\
\hline Never smokers & $22(20.4)$ & $12(21.8)$ & $21(20.2)$ & \\
\hline \multicolumn{5}{|l|}{ Time since first diagnosis yrs } \\
\hline$<1$ & $38(35.2)$ & $20(36.4)$ & $41(39.4)$ & 0.86 \\
\hline $1-3$ & $29(26.9)$ & $13(23.6)$ & $25(24.0)$ & \\
\hline Current steroid use & $8(7.4)$ & $6(10.9)$ & $5(4.8)$ & \\
\hline Surgical lung biopsy & $26(24.1)$ & $16(29.1)$ & $28(26.9)$ & 0.78 \\
\hline VC $\mathrm{mL}$ & $2400.8 \pm 638.4$ & $2437.8 \pm 684.8$ & $2472.3 \pm 698.9$ & 0.74 \\
\hline VC $\%$ pred & $77.3 \pm 16.8$ & $76.2 \pm 18.7$ & $79.1 \pm 17.4$ & 0.57 \\
\hline TLC \% pred & $73.2 \pm 16.5$ & $72.4 \pm 15.6$ & $75.2 \pm 15.7$ & 0.50 \\
\hline DL,co \% pred & $52.1 \pm 16.8$ & $53.6 \pm 19.1$ & $55.2 \pm 18.2$ & 0.44 \\
\hline $\mathrm{Pa}, \mathrm{O}_{2}$ at rest $\mathrm{mmHg}$ & $79.8 \pm 10.2$ & $81.6 \pm 8.4$ & $81.0 \pm 9.5$ & 0.48 \\
\hline $\mathrm{PA}-\mathrm{a}, \mathrm{O}_{2} \mathrm{mmHg}$ & $18.4 \pm 11.3$ & $16.9 \pm 9.6$ & $17.4 \pm 9.7$ & 0.64 \\
\hline Lowest $\mathrm{Sp}, \mathrm{O}_{2} \%$ & $89.0 \pm 2.3$ & $88.8 \pm 2.4$ & $89.0 \pm 2.0$ & 0.86 \\
\hline Presence of desaturation below $88 \%$ on walk test & $34(31.5)$ & $19(34.5)$ & $24(23.1)$ & \\
\hline
\end{tabular}

Data are presented as $n, n(\%)$ or mean $\pm \mathrm{SD}$, unless stated otherwise. VC: vital capacity; \% pred: \% predicted; TLC: total lung capacity; $D L, C O$ : diffusion capacity of the lung for carbon monoxide; $\mathrm{Pa}_{\mathrm{a}} \mathrm{O}_{2}$ : arterial oxygen tension; $\mathrm{PA}_{\mathrm{A}} \mathrm{a}_{2}$ : alveolar-arterial oxygen tension difference; $\mathrm{Sp}, \mathrm{O}_{2}$ : oxygen saturation measured by pulse oximetry.

\#: using Fisher's exact test, Kruskal-Wallis test and ANCOVA in accordance with nominal and binary, ordinal and continuous data, respectively. VC, TLC, DL,CO, $P a, O_{2}$ and $P \mathrm{~A}-\mathrm{a}, \mathrm{O}_{2}$ were measured for 106 subjects in the high-dose pirfenidone group, and TLC and $\mathrm{DL}, \mathrm{CO}$ for 103 subjects in the placebo group. ${ }^{\bullet} \mathrm{p}<0.15$.

\section{Effects on secondary and tertiary end-points}

The secondary end-points were PFS time and the change in the lowest $\mathrm{Sp}, \mathrm{O}_{2}$ during the 6MET. The distribution of PFS time was compared between the high-dose and placebo groups with the log-rank test, and a significant difference was found $(\mathrm{p}=0.0280$; fig. 4). In addition, a marginally significant difference was found in the distribution between the low-dose and placebo groups $(p=0.0655)$. No statistically significant difference was detected in the mean changes of the lowest $S \mathrm{p}, \mathrm{O}_{2}$ among the three groups (table 4 ). The incidence of acute exacerbation during the study or within 28 days after the termination of the study was six $(5.6 \%)$, three $(5.5 \%)$ and five $(4.8 \%)$ in the high-dose, low-dose and placebo groups, respectively. No significant differences were seen among the three groups. Although between the low-dose and the placebo groups the differences of mean changes in TLC and changes in DL,CO were statistically significant $(p=0.0408$ and $p=0.0768$, respectively) at week 52 , there were no significant differences in the changes of other PFTs or serum markers among the three groups (table E1 in the online supplementary material).

\section{Compliance and safety}

Significant adverse events reported with a frequency of $\geqslant 5 \%$ during the study $(\mathrm{p}<0.05)$ are listed in table 5. Photosensitivity, anorexia, dizziness and elevated $\gamma$-glutamyl-transpeptidase $(\gamma$ GTP) were significantly more common in the high-dose group than in the placebo group, and photosensitivity, asteatotic eczema, abdominal discomfort and decrease in white blood cells were significantly more common in the low-dose group than in the placebo group. In contrast, respiratory tract infection, such as nasopharyngitis and upper respiratory tract inflammation, was significantly less common in the high-dose group than the placebo group.

The adverse events leading to discontinuation of the study are listed in table 2. $20(18.3 \%)$ patients in the high-dose group and $11(20 \%)$ in the low-dose group discontinued the study treatment, with no statistical differences compared to 14 $(13.1 \%)$ patients in the placebo group. Most of the adverse events disappeared with a decrease in the dose or temporary withholding of the medication. Therefore, treatment with pirfenidone was generally well tolerated in patients with IPF.

Photosensitivity was the major adverse event observed in 51\% of the patients in the high-dose group and 53\% in low-dose group. Of the patients who developed photosensitivity, $\sim 70 \%$ and $80 \%$ in the high-dose and the low-dose groups, respectively, were mild cases and the remainder were moderate cases. Although there were no significant differences in the 


\begin{tabular}{|c|c|c|c|c|}
\hline TABLE 2 & \multicolumn{4}{|c|}{$\begin{array}{l}\text { Reasons for discontinuing the study (full analysis } \\
\text { set) }\end{array}$} \\
\hline \multicolumn{2}{|c|}{ Reasons for discontinuation } & High dose & Low dose & Placebo \\
\hline \multicolumn{2}{|l|}{ Subjects } & 108 & 55 & 104 \\
\hline \multicolumn{2}{|l|}{ Total } & $40(37.0)$ & $15(27.3)$ & $31(29.8)$ \\
\hline \multicolumn{2}{|c|}{ Progression of disease } & $8(7.4)$ & $0(0.0)$ & $15(14.4)$ \\
\hline \multicolumn{2}{|l|}{ Death } & $0(0.0)$ & $0(0.0)$ & $0(0.0)$ \\
\hline \multicolumn{2}{|c|}{$10 \%$ decline in VC } & $5(4.6)$ & $0(0.0)$ & $11(10.6)$ \\
\hline \multicolumn{2}{|c|}{ Worsening of respiratory symptoms } & $3(2.8)$ & $0(0.0)$ & $4(3.7)$ \\
\hline \multicolumn{2}{|c|}{ Acute exacerbation } & $4(3.7)$ & $2(3.6)$ & $4(3.8)$ \\
\hline \multicolumn{2}{|c|}{ Adverse events ${ }^{\#}$} & $15(13.9)$ & $9(16.4)$ & $7(6.7)$ \\
\hline \multicolumn{2}{|c|}{ Photosensitivity } & $3(2.8)$ & $2(3.6)$ & $0(0.0)$ \\
\hline \multicolumn{2}{|c|}{ Lung carcinoma } & $2(1.8)$ & $1(1.8)$ & $1(0.9)$ \\
\hline \multicolumn{2}{|l|}{ Fever } & $2(1.8)$ & $0(0.0)$ & $0(0.0)$ \\
\hline \multicolumn{2}{|c|}{ Respiratory failure } & $2(1.8)$ & $0(0.0)$ & $0(0.0)$ \\
\hline \multicolumn{2}{|l|}{ Rash } & $1(0.9)$ & $1(1.8)$ & $0(0.0)$ \\
\hline \multicolumn{2}{|c|}{ AST and/or ALT increase } & $1(0.9)$ & $1(1.8)$ & $0(0.0)$ \\
\hline \multicolumn{2}{|c|}{ Gastric ulcer } & $1(0.9)$ & $0(0.0)$ & $0(0.0)$ \\
\hline \multicolumn{2}{|l|}{ Anorexia } & $1(0.9)$ & $0(0.0)$ & $0(0.0)$ \\
\hline \multicolumn{2}{|c|}{ Muscle pain } & $1(0.9)$ & $0(0.0)$ & $0(0.0)$ \\
\hline \multicolumn{2}{|l|}{ Dysgeusia } & $1(0.9)$ & $0(0.0)$ & $0(0.0)$ \\
\hline \multicolumn{2}{|c|}{ Loss of consciousness } & $1(0.9)$ & $0(0.0)$ & $0(0.0)$ \\
\hline \multicolumn{2}{|c|}{ Respiratory tract infection } & $0(0.0)$ & $2(3.6)$ & $0(0.0)$ \\
\hline \multicolumn{2}{|c|}{ Fatigue, drowsiness } & $0(0.0)$ & $1(1.8)$ & $0(0.0)$ \\
\hline \multicolumn{2}{|c|}{ Rheumatoid arthritis } & $0(0.0)$ & $1(1.8)$ & $0(0.0)$ \\
\hline \multicolumn{2}{|c|}{ Electrocardiogram abnormal } & $0(0.0)$ & $0(0.0)$ & $2(1.9)$ \\
\hline \multicolumn{2}{|l|}{ Nausea } & $0(0.0)$ & $0(0.0)$ & $1(0.9)$ \\
\hline \multicolumn{2}{|c|}{ Lung neoplasm } & $0(0.0)$ & $0(0.0)$ & $1(0.9)$ \\
\hline \multicolumn{2}{|c|}{ MPO-ANCA increase } & $0(0.0)$ & $0(0.0)$ & $1(0.9)$ \\
\hline \multicolumn{2}{|c|}{ Cerebral thrombosis } & $0(0.0)$ & $0(0.0)$ & $1(0.9)$ \\
\hline Suicide & & $0(0.0)$ & $0(0.0)$ & $1(0.9)$ \\
\hline Upper resp & atory tract infection & $0(0.0)$ & $0(0.0)$ & $1(0.9)$ \\
\hline Pneumotho & & $0(0.0)$ & $0(0.0)$ & $1(0.9)$ \\
\hline Consent wit & drawn & $12(11.1)$ & $2(3.6)$ & $4(3.8)$ \\
\hline Other & & $1(0.9)$ & $2(3.6)$ & $1(1.0)$ \\
\hline
\end{tabular}

Data are presented as $n$ or $n(\%)$. VC: vital capacity; AST: aspartate aminotransferase; ALT: alanine aminotransferase: MPO-ANCA: myeloperoxidase-

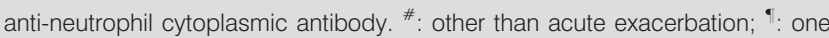
case was respiratory failure due to traumatic shock caused by a traffic accident.

incidence between the high-dose and low-dose groups, the percentage of mild photosensitivity was higher in the low-dose group. The assessment of the degree of the severity was subjective, based on the patient's symptoms and the site investigator's judgement. Only three patients $(\sim 3 \%)$ discontinued the study due to photosensitivity.

\section{DISCUSSION}

During the last decade, several clinical trials for IPF have been conducted worldwide to determine an effective treatment regimen for IPF, but the results have been negative and disappointing. Thus, an effective treatment regimen compared with placebo controls is yet to be determined [5, 6, 21, 28]. In this trial, both high- and low-dose pirfenidone groups improved VC, and the distribution of PFS was better than in the placebo group (fig. 2, table 3, fig. 4). Recent studies have

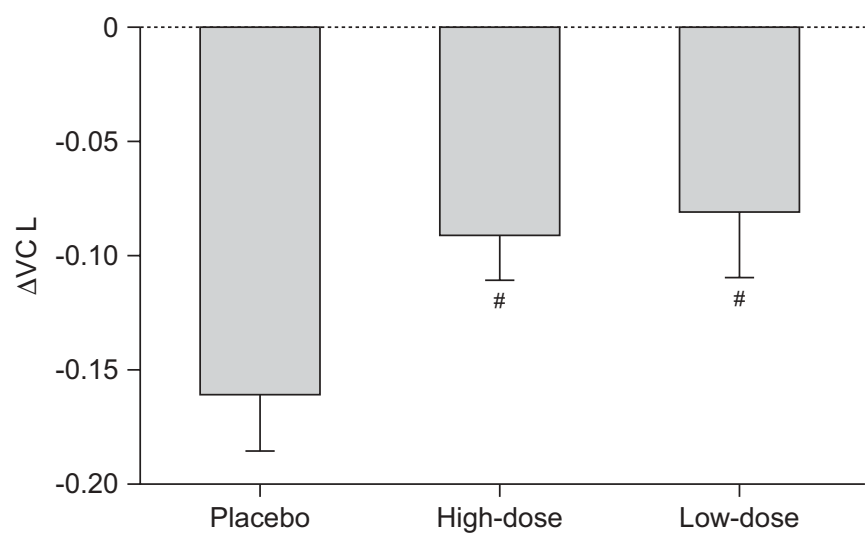

FIGURE 2. Effects of pirfenidone on vital capacity (VC) at week 52. Data are presented as mean $\pm \mathrm{SE}$. ${ }^{*}: \mathrm{p}<0.1$, comparison of adjusted means based on ANCOVA (negative and positive of the changes represent deterioration and improvement from baseline, respectively). The last observation carried forward method was used for drop-outs in each group. Placebo group: $n=103$; high-dose group: $n=104$; low-dose group: $n=54$.

confirmed that a fall in VC or forced vital capacity (FVC) of $\geqslant 10 \%$ from the baseline over a period of $6-12$ months is the most important predictor of mortality in patients with IPF [22$24,26]$. Therefore, disease progression, defined as time to death and/or $\geqslant 10 \%$ decline in absolute changes in measured VC, is acknowledged as an appropriate surrogate marker for survival $[6,23]$ that is also appropriate regarding lesser changes in FVC reported recently [29]. Considering that $>90 \%$ of patients had not received any treatment prior to randomisation, our findings provide first evidence that a treatment intervention with a drug improves PFS time in patients with IPF.

No significant differences were found with respect to the changes in the lowest $\mathrm{Sp}, \mathrm{O}_{2}$ among the groups in our study (table 4). While the exact reasons for these apparent negative observations are unknown, the following facts may explain our observations relating to the discrepancy in findings between the previous study [15] and the present one: 1) the 6MET performed in this and the previous study is not a validated test, and 2) the final change in the lowest $S p, \mathrm{O}_{2}$ could not be accurately evaluated because $\sim 20 \%$ of the patients could not complete the 6MET during follow-up as their lowest $S \mathrm{p}, \mathrm{O}_{2}$ had reached $82 \%$ (data not shown). Reproducibility of the exercise studies with the 6MWT and modified versions of the 6MWT/ $6 \mathrm{MET}$ are confounding factors that need to be clarified for future studies before embarking on exercise studies such as the 6MET/6MWT $[6,28]$.

The previous phase II trial in Japan was terminated early because of the incidence of acute exacerbations of IPF [15]. However, in the present trial, no differences were found in the frequency of acute exacerbation among the three groups. While the incidence of acute exacerbation in the placebo group was $14.3 \%$ over 9 months in the previous study, this was observed in only $4.8 \%$ over 52 weeks in the current study, and, thus, the previous observation was not confirmed in the present study. The reasons for this discrepancy are unclear. In the present study, acute exacerbation occurred in only $5 \%$ of the patients with relatively mild pulmonary function impairment during 


\begin{tabular}{|c|c|c|c|c|c|c|c|c|}
\hline High dose & $2.40 \pm 0.64$ & 106 & $2.36 \pm 0.73$ & 67 & 104 & $-0.09 \pm 0.02$ & $0.07 \pm 0.03$ & 0.0416 \\
\hline Placebo & $2.47 \pm 0.70$ & 104 & $2.42 \pm 0.75$ & 72 & 103 & $-0.16 \pm 0.02$ & & \\
\hline
\end{tabular}

$1 \mathrm{yr}$. The true incidence and prevalence of the acute exacerbation of IPF is unknown; the frequencies of acute exacerbation have been reported to differ among studies, which are largely retrospective [30]. Nevertheless, our observations regarding acute exacerbation warrant further studies to carefully assess the problem in a well-defined, larger study with longer followup data.

The post hoc analysis based on respiratory function categories was carried out in this study to compare the results from our previous phase II study [15]. The improvement ratings of each respiratory function were defined by ATS criteria. There was no significant difference between the high-dose group and the placebo group (see online supplementary material, Fig. E2-1).
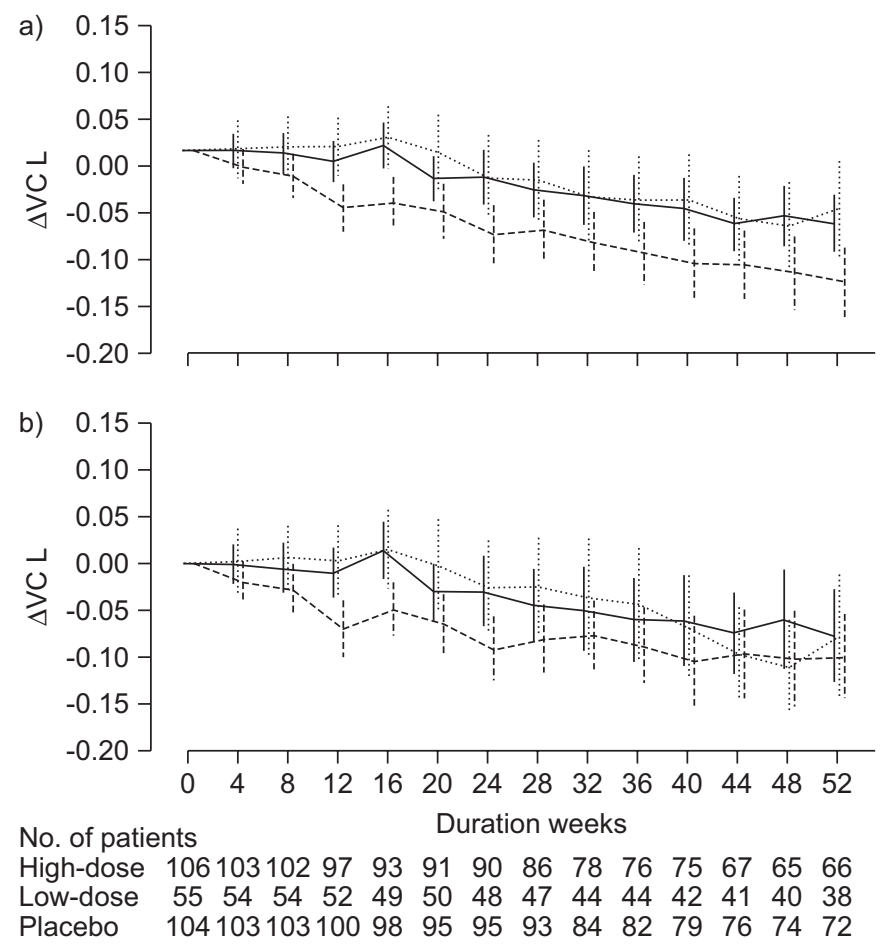

FIGURE 3. The serial changes in vital capacity (VC) every 4 weeks over 52 weeks. a) Transitional plots of "last observation carried forward imputed means". b) "Crude means" of the changes in VC. Data are presented as mean $\pm 90 \% \mathrm{Cl}$. —_ : high-dose pirfenidone group; …....... low-dose pirfenidone group; ----: placebo group.
The reasons for the differences between the two studies are unknown. However, the difference between the high-dose group and the placebo group was significant $(p=0.0053)$ when the categorised analysis of the changes in VC was based on the rating of a lesser magnitude (see online supplementary material, Fig. E2-2) [29].

The adverse event that occurred significantly more often among patients in both the high- and low-dose pirfenidone groups was photosensitivity, a well-known side-effect associated with pirfenidone that has been documented in previous studies [7, 15]. Anorexia and elevated $\gamma$-GTP were significantly more common in the high-dose group than in the placebo group, similar to results observed in our phase II study [15]. Although the overall incidence of adverse events in the pirfenidone treatment groups was relatively high, no significant differences were detected in the frequency of the patients who discontinued the study between the pirfenidone treatment groups and the placebo group. This may be in part that the patients were well informed regarding the side-effect of rashes. Despite the manifestation of the anticipated skin rash, pirfenidone was generally well tolerated in IPF patients.

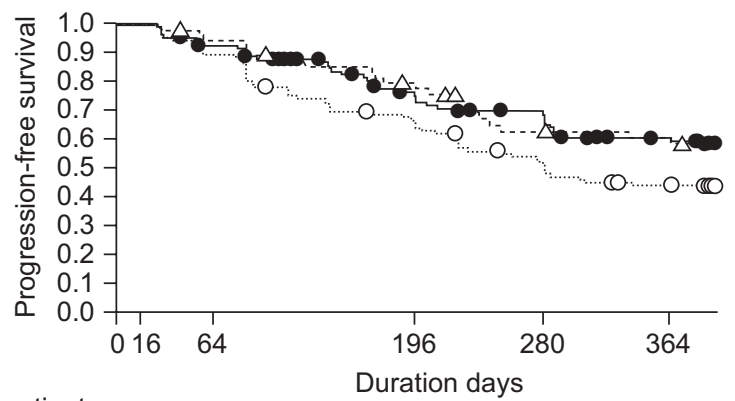

No. of patients

$\begin{array}{lllllll}\text { High-dose } & 106 & 106 & 95 & 69 & 59 & 45 \\ \text { Low-dose } & 55 & 55 & 51 & 39 & 29 & 26\end{array}$

Low-dose $55 \quad 55 \quad 51$

Placebo 10410493

66

FIGURE 4. Kaplan-Meier plot of progression-free survival time among idiopathic pulmonary fibrosis patient groups. - - : high dose; ----: low dose; .........: placebo. Symbols on the curve represent the censored points where patients discontinued the study treatment due to causes other than progression of the disease. Kaplan-Meier curves were compared with the log-rank test: $p=0.0280$ between the high-dose group and placebo group; $p=0.0655$ between the low-dose group and placebo group; $p=0.9106$ between the high-dose group and low-dose group. 
TABLE 4 Comparison of changes in the lowest arterial oxygen saturation measured by pulse oximetry

\begin{tabular}{|c|c|c|c|c|}
\hline Group & Subjects $\mathrm{n}$ & Adjusted mean $\pm \mathrm{SE}$ & Mean \pm SE difference from placebo group $\%$ & p-value $\#$ \\
\hline High-dose & 99 & $-1.70 \pm 0.35$ & $-0.17 \pm 0.50$ & 0.7393 \\
\hline Placebo & 100 & $-1.53 \pm 0.35$ & & \\
\hline
\end{tabular}

Potential limitations of this study include changing the primary end-point during the study. Despite the evolved knowledge that the change in VC at 12 months correlated well with survival $[22-24,26]$, we had initially chosen the lowest $S p, \mathrm{O}_{2}$ during the $6 \mathrm{MET}$ as the primary end-point for this study as we had been encouraged by the novel observations in our previous study [15]. Change in VC was initially intended to be a secondary endpoint. Acknowledging that the 6MET employed in our phase II study needed to be validated, a validation study was planned to evaluate the lowest $S_{\mathrm{p}}, \mathrm{O}_{2}$ during the $6 \mathrm{MET}$ prior to the initiation of the phase III study (research supported by health and labour sciences research grants). VC and PFS were selected as key secondary end-points to support the primary end-point, and the power of test for VC and PFS was based on the sample size calculated from the lowest $\mathrm{Sp}_{\mathrm{p}} \mathrm{O}_{2}$ in the first version of the protocol itself. However, significant difficulties were confronted during the validation study for the 6MET and several patients dropped out of the phase III study. Because of the concerns of assessing the efficacy of pirfenidone based on the lowest $\mathrm{Sp}, \mathrm{O}_{2}$ measured every 12 weeks compared with the VC measured every 4 weeks and of the potential for unexpectedly large fluctuations between each point in the lowest $S_{\mathrm{p}}, \mathrm{O}_{2}$ along with the problems of reliability/reproducibility of the $S p, \mathrm{O}_{2}$ measurement during exertion such as walking [27] and acknowledging that the change in VC or FVC was increasingly being used as the primary end-point in other clinical studies $[5,21]$, the primary end-point was changed from the lowest $\mathrm{Sp}, \mathrm{O}_{2}$ during the $6 \mathrm{MET}$ to VC during the study period. While this change in the primary end-point may be considered as a major limitation for this study, it should be noted that the decision to change the endpoint was prior to code-breaking according to the recommendation of the independent DSMB, and the sample size was unaffected.

We acknowledge the limitations of dealing with missing values. It is generally known that results of analyses may have potential bias when missing values are imputed by an arbitrary method, and that there is no perfect imputation method which performs best under all circumstances. In this study, we adopted LOCF, since LOCF had been adopted in the previous study. We were under the impression that LOCF would not tip the balance in favour of either of the treatment groups, if there were no substantial differences in the rate of drop-outs. The mixed model approach using repeated measures of changes in VC without LOCF imputation as a sensitivity analysis also showed significant or marginally significant treatment effects and supported the LOCF analysis. Figure 3 shows the transitional plot of the changes in VC over 52 weeks. Both

TABLE 5 Significant adverse events ${ }^{\#}$

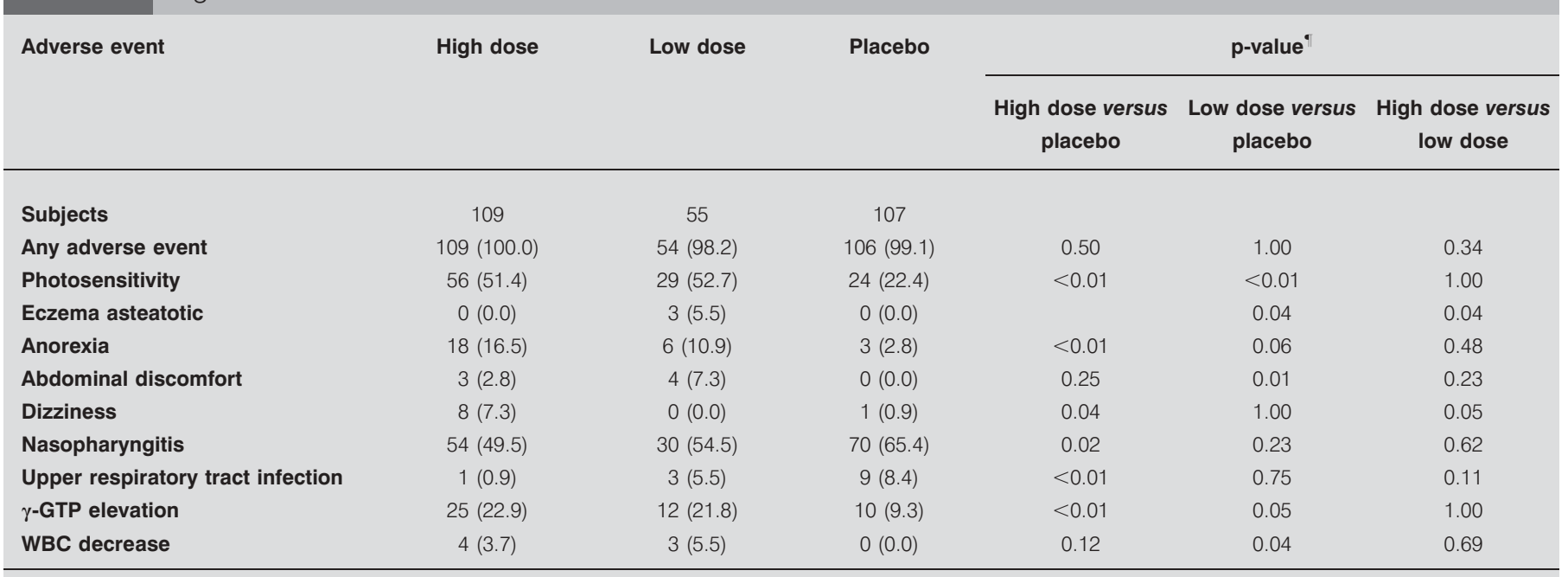

Data are presented as $\mathrm{n}$ or $\mathrm{n}(\%)$, unless otherwise stated. ${ }^{\#}$ : events which were observed with an incidence of $\geqslant 5 \%$ during the study period and for which a significant difference was detected between the placebo group and each of the pirfenidone treatment groups, high dose or low dose $(p<0.05)$; ${ }^{\circ}$ : using Fisher's exact test. $\gamma$-GTP: $\gamma$ glutamyl-transpeptidase; WBC: white blood cell. 
the "LOCF imputed means" and "crude means" of the changes within 16 weeks suggested a favourable effect of pirfenidone and were not affected by drop-outs.

Other potential limitations of our study include the following. 1) A selection bias, as patients enrolled in this study needed to be able to perform the $6 \mathrm{MET}$ at baseline in accordance with the protocol; the results in this selected group of patients with mild functional impairment may not therefore be applicable to all patients with IPF with varying degrees of pulmonary symptoms and functional impairment. 2) The lack of a central pathology review. While we acknowledge these limitations, it must be noted that the patient population enrolled in this study included all consecutive, eligible and consenting patients from the general patient population with IPF with mild functional impairment.

In conclusion, the results of the phase III clinical trial demonstrate that pirfenidone, a novel antifibrotic agent, preserves VC and improves PFS better than placebo in Japanese patients with IPF with mild functional impairment without serious adverse events. Future studies may confirm our findings further.

\section{SUPPORT STATEMENT}

This work was supported by a grant-in-aid for and by members of interstitial lung diseases from the Japanese Ministry of Health, Labor and Welfare, and also by members of the Japanese Respiratory Society's committee for diffuse lung diseases.

\section{CLINICAL TRIAL}

This clinical trial was registered with the Japan Pharmaceutical Information Center (JAPIC) on September 13, 2005 (registration number: JAPICCTCI-050121).

\section{STATEMENT OF INTEREST}

Statements of interest for H. Taniguchi, M. Ebina, T. Ogura, A. Azuma, M. Suga, Y. Taguchi, H. Takahashi, K. Nakata, A. Sato, M. Takeuchi, G. Raghu, S. Kudoh and T. Nukiwa, and for the study itself can be found at www.erj.ersjournals.com $/ \mathrm{misc} /$ statements.dtl

\section{ACKNOWLEDGEMENTS}

The authors would like to thank: M. Ando (Omotesando Yoshida Hospital, Kumamoto, Japan), S. Kitamura (Minami-Tochigi Hospital, Oyama, Tochigi, Japan), Y. Nakai (Tanpopo Clinic, Sendai, Miyagi, Japan) and A. Kondo (Niigata Tetsudo Kensin Center, Niigata, Niigata, Japan) of the independent DSMB; and K. Murata (Shiga University of Medical Science Hospital, Ohtsu, Shiga, Japan), M. Takahashi (Shiga University of Medical Science Hospital), H. Hayashi (Japanese Red Cross Okayama Hospital, Okayama, Japan), S. Noma (Tenri Hospital, Tenri, Nara, Japan), T. Johkoh (Osaka University Hospital, Osaka, Japan), H. Arakawa (Dokkyo Medical University Hospital, Shimotuga, Tochigi, Japan) and K. Ichikado (Kumamoto University Hospital, Kumamoto, Japan) of the Imaging Central Judging Panel. The authors are also grateful to E. Tsuboi (Toranomon Hospital, Minato, Tokyo, Japan) for his expert advice on the 6-min steady-state exercise test. Finally, thanks are extended to M. Igarashi, T. Fukunaga, T. Kitai, T. Tokura, Y. Tsuchiya, S. Kakutani, H. Oku and Y. Tsukamoto (all Shionogi \& Co. Ltd, Osaka, Japan) for their advice and for reviewing the manuscript.

The members of the Pirfenidone Clinical Study Group in Japan are as follows. T. Betsuyaku (Hokkaido University Hospital, Sapporo, Hokkaido). Y. Sugawara (Kyowakai Obihiro Respiratory Hospital, Obihiro, Hokkaido). S. Fujiuchi (Dohoku National Hospital,
Asahikawa, Hokkaido). K. Yamauchi (Iwate Medical University Hospital, Morioka, Iwate). K. Konishi (Morioka Tsunagi Onsen Hospital, Morioka). M. Munakata (Fukushima Medical University Hospital, Fukushima). Y. Kimura (Tohoku University Hospital, Sendai, Miyagi). Y. Ishii (Dokkyo Medical University Hospital, Shimotsuga, Tochigi). Y. Sugiyama (Jichi Medical University Hospital, Shimotsuke, Tochigi). K. Kudoh (International Medical Center of Japan, Shinjuku, Tokyo). T. Saito (Ibarakihigashi National Hospital, Naka, Ibaraki). T. Yamaguchi (JR Tokyo General Hospital, Shibuya, Tokyo). A. Mizoo (Tokyo Kosei Nenkin Hospital, Shinjuku). A. Nagai (Tokyo Women's Medical University Hospital, Shinjuku). A. Ishizaka and K. Yamaguchi (Keio University Hospital, Shinjuku). K. Yoshimura (Toranomon Hospital, Minato, Tokyo). M. Oritsu (Japanese Red Cross Medical Center, Shibuya). Y. Fukuchi and K. Takahashi (Juntendo University Hospital, Bunkyo, Tokyo). K. Kimura (Toho University Omori Medical Center, Ota, Tokyo). Y. Yoshizawa (Tokyo Medical and Dental University Hospital, Bunkyo). T. Nagase (Tokyo University Hospital, Bunkyo). T. Hisada (Tokyo Teishin Hospital, Chiyoda, Tokyo). K. Ohta (Teikyo University Hospital, Itabashi, Tokyo). K. Yoshimori (Fukujuji Hospital, Kiyose, Tokyo). Y. Miyazawa and K. Tatsumi (Chiba University Hospital, Chiba). Y. Sasaki (Chiba-East Hospital, Chiba). M. Taniguchi (Sagamihara National Hospital, Sagamihara, Kanagawa). Y. Sugita (Saitama Cardiovascular and Respiratory Center, Kumagaya, Saitama). E. Suzuki (Niigata University Medical \& Dental Hospital, Niigata). Y. Saito (Nishi-Niigata Chuo National Hospital, Niigata). H. Nakamura (Seirei Hamamatsu General Hospital, Hamamatsu, Shizuoka). K. Chida (Hamamatsu University School of Medicine, University Hospital, Hamamatsu). N. Kasamatsu (Hamamatsu Medical Center, Hamamatsu). H. Hayakawa (Tenryu Hospital, Hamamatsu, Shizuoka). K. Yasuda (Iwata City Hospital, Iwata, Shizuoka). H. Suganuma (Shimada Municipal Hospital, Shimada, Shizuoka). H. Genma (Fukuroi Municipal Hospital, Fukuroi, Shizuoka). R. Tamura (Fujieda Municipal General Hospital, Fujieda, Shizuoka). T. Shirai (Fujinomiya City General Hospital, Fujinomiya, Shizuoka). J. Shindoh (Ogaki Municipal Hospital, Ogaki, Gifu). S. Sato (Nagoya City University Hospital, Nagoya, Aichi). O. Taguchi (Mie University Hospital, Tsu, Mie). Y. Sasaki (Kyoto Medical Center, Fushimi, Kyoto), H. Ibata (Mie Chuo Medical Center, Tsu). M. Yasui (Kanazawa University Hospital, Kanazawa, Ishikawa). Y. Nakano (Shiga Medical University Hospital, Otsu, Shiga). M. Ito and S. Kitada (Toneyama National Hospital, Toyonaka, Osaka). H. Kimura (Nara Medical University Hospital, Kashihara, Nara). Y. Inoue (Kinki-Chuo Chest Medical Center, Sakai, Osaka). H. Yasuba (Takatsuki Red Cross Hospital, Takatsuki, Osaka). Y. Mochizuki (Himeji Medical Center, Himeji, Hyogo). S. Horikawa and Y. Suzuki (Japanese Red Cross Wakayama Medical Centre, Wakayama). N. Katakami (Institute of Biomedical Research and Innovation Hospital, Kobe, Hyogo). Y. Tanimoto (Okayama University Hospital, Okayama). Y. Hitsuda and N. Burioka (Tottori University Hospital, Yonago, Tottori). T. Sato (Okayama Medical Center, Okayama). N. Kohno and A. Yokoyama (Hiroshima University Hospital, Hiroshima). Y. Nishioka (Tokushima University Hospital, Tokushima). N. Ueda (Ehime Prefectural Central Hospital, Matsuyama, Ehime). K. Kuwano (Kyusyu University Hospital, Fukuoka). K. Watanabe (Fukuoka University Hospital, Fukuoka). H. Aizawa (Kurume University Hospital, Kurume, Fukuoka). S. Kohno and H. Mukae (Nagasaki University Hospital of Medicine and Dentistry, Nagasaki). H. Kohrogi (Kumamoto University Hospital, Kumamoto). J. Kadota, I. Tokimatsu and E. Miyazaki (Oita University Hospital, Yufu, Oita). T. Sasaki (Miyazaki University Hospital, Miyazaki). M. Kawabata (Minami Kyusyu National Hospital, Aira, Kagoshima).

\section{REFERENCES}

1 Bjoraker JA, Ryu JH, Edwin MK, et al. Prognostic significance of histopathologic subsets in idiopathic pulmonary fibrosis. Am J Respir Crit Care Med 1998; 157: 199-203. 
2 Schwartz DA, Helmers RA, Galvin JR, et al. Determinants of survival in idiopathic pulmonary fibrosis. Am J Respir Crit Care Med 1994; 149: 450-454.

3 Crystal RG, Bitterman PB, Mossman B, et al. Future research directions in idiopathic pulmonary fibrosis: summary of a National Heart, Lung, and Blood Institute Working Group. Am J Respir Crit Care Med 2002; 166: 236-246.

4 Selman M, Pardo A. Role of epithelial cells in idiopathic pulmonary fibrosis: from innocent targets to serial killers. Proc Am Thorac Soc 2006; 3: 364-372.

5 Raghu G, Brown KK, Bradford WZ, et al. A placebo-controlled trial of interferon- $\gamma-1 \mathrm{~b}$ in patients with idiopathic pulmonary fibrosis. N Engl J Med 2004; 350: 125-133.

6 King TE Jr, Behr J, Brown KK, et al. BUILD-1: a randomized placebo-controlled trial of bosentan in idiopathic pulmonary fibrosis. Am J Respir Crit Care Med 2008; 177: 75-81.

7 Raghu G, Johnson WC, Lockhart D, et al. Treatment of idiopathic pulmonary fibrosis with a new antifibrotic agent, pirfenidone: results of a prospective, open-label phase II study. Am J Respir Crit Care Med 1999; 159: 1061-1069.

8 Gahl WA, Brantly M, Troendle J, et al. Effect of pirfenidone on the pulmonary fibrosis of Hermansky-Pudlak syndrome. Mol Genet Metab 2002; 76: 234-242.

9 Nagai S, Hamada K, Shigematsu M, et al. Open-label compassionate use one year-treatment with pirfenidone to patients with chronic pulmonary fibrosis. Intern Med 2002; 41: 1118-1123.

10 Iyer SN, Gurujeyalakshmi G, Giri SN. Effects of pirfenidone on transforming growth factor- $\beta$ gene expression at the transcriptional level in bleomycin hamster model of lung fibrosis. J Pharmacol Exp Ther 1999; 291: 367-373.

11 Gurujeyalakshmi G, Hollinger MA, Giri SN. Pirfenidone inhibits PDGF isoforms in bleomycin hamster model of lung fibrosis at the translational level. Am J Physiol 1999; 276: L311-L318.

12 Iyer SN, Gurujeyalakshmi G, Giri SN. Effects of pirfenidone on procollagen gene expression at the transcriptional level in bleomycin hamster model of lung fibrosis. J Pharmacol Exp Ther 1999; 289: 211-218.

13 Misra HP, Rabideau C. Pirfenidone inhibits NADPH-dependent microsomal lipid peroxidation and scavenges hydroxyl radicals. Mol Cell Biochem 2000; 204: 119-126.

$14 \mathrm{Oku} \mathrm{H}$, Shimizu $\mathrm{T}$, Kawabata $\mathrm{T}$, et al. Antifibrotic action of pirfenidone and prednisolone: Different effects on pulmonary cytokines and growth factors in bleomycininduced murine pulmonary fibrosis. Eur J Pharmacol 2008; 590: 400-408.

15 Azuma A, Nukiwa T, Tsuboi E, et al. Double-blind, placebocontrolled trial of pirfenidone in patients with idiopathic pulmonary fibrosis. Am J Respir Crit Care Med 2005; 171: 1040-1047.
16 American Thoracic Society. Idiopathic pulmonary fibrosis: diagnosis and treatment: international consensus statement. American Thoracic Society (ATS), and the European Respiratory Society (ERS). Am J Respir Crit Care Med 2000; 161: 646-664.

17 Japanese Respiratory Society's Committee formulating diagnosis and treatment guideline for diffuse lung diseases. Clinical diagnostic and treatment guidance for idiopathic interstitial pneumonias. Tokyo, Nankodo, 2004; pp. 63-65.

18 Taves DR. Minimization: a new method of assigning patients to treatment and control groups. Clin Pharmacol Ther 1974; 15: 443-453.

19 Efron B. Randomizing and balancing a complicated sequential experiment. In: Miller RG, Efron B, Brown BW, Moses LE, eds. The Biostatistics Casebook. New York, Wiley, 1980; pp. 19-30.

20 Taniguchi H, Kondoh Y. Revised criteria for acute exacerbation of idiopathic pulmonary fibrosis. The Annual Report by Study Group of Ministry of Health and Welfare for Diffuse Lung Disease. Diffiuse Lung Diseases Research Group from the Ministry of Health, Labor and Welfare of Japanese Government, 2004; pp. 114-119.

21 Demedts M, Behr J, Buhl R, et al. High-dose acetylcysteine in idiopathic pulmonary fibrosis. N Engl J Med 2005; 353: 2229-2242.

22 Flaherty KR, Mumford JA, Murray S, et al. Prognostic implications of physiologic and radiographic changes in idiopathic interstitial pneumonia. Am J Respir Crit Care Med 2003; 168: 543-548.

23 King TE Jr, Safrin S, Starko KM, et al. Analyses of efficacy end points in a controlled trial of interferon- $\gamma 1 \mathrm{~b}$ for idiopathic pulmonary fibrosis. Chest 2005; 127: 171-177.

24 Latsi PI, du Bois RM, Nicholson AG, et al. Fibrotic idiopathic interstitial pneumonia: The prognostic value of longitudinal functional trends. Am J Respir Crit Care Med 2003; 168: 531-537.

25 King TE Jr, Tooze JA, Schwarz MI, et al. Predicting survival in idiopathic pulmonary fibrosis: scoring system and survival model. Am J Respir Crit Care Med 2001; 164: 1171-1181.

26 Collard HR, King TE Jr, Bartelson BB, et al. Changes in clinical and physiologic variables predict survival in idiopathic pulmonary fibrosis. Am J Respir Crit Care Med 2003; 168: 538-542.

27 Eaton T, Young P, Milne D, et al. Six-minute walk, maximal exercise tests. Reproducibility in fibrotic interstitial pneumonia. Am J Respir Crit Care Med 2005; 171: 1150-1157.

28 Raghu G, Brown KK, Costabel U, et al. Treatment of idiopathic pulmonary fibrosis with etanercept: An exploratory, placebocontrolled trial. Am J Respir Crit Care Med 2008; 178: 948-955.

29 Zappala CJ, Latsi PI, Nicholson AG, et al. Marginal decline in forced vital capacity is associated with a poor outcome in idiopathic pulmonary fibrosis. Eur Respir J 2010; 35: 830-835.

30 Collard HR, Moore BB, Flaherty KR, et al. Acute exacerbations of idiopathic pulmonary fibrosis. Am J Respir Crit Care Med 2007; 176: 636-643. 